\title{
Adenoma ectópico hipofisiario: Reporte de un caso y revisión de la literatura
}

\section{Ectopic hypophysial adenoma: Case report and literature review}

\author{
Ricardo Quitral C', José Miguel Contreras R².
}

\begin{abstract}
RESUMEN
Los adenomas son los tumores hipofisiarios más frecuentes y ocasionalmente se pueden ubicar fuera de la silla turca, ya sea por extensión desde ésta o más rara vez por ubicación ectópica.

Se presenta el caso de una paciente de 56 años, en la que en un TAC de cerebro solicitado en el contexto de un síndrome vertiginoso, se encuentra una lesión en región esfenoidal que se extiende al clivus, frente a la cual se plantean como posibilidades diagnósticas: cordoma, carcinoma nasofaríngeo, tumor derivado de una glándula salival menor y un tumor metastático. La lesión es abordada y extraída endoscópicamente, comprobándose finalmente que correspondía a un adenoma ectópico hipofisiario.
\end{abstract}

Palabras clave: Adenoma ectópico, Hipófisis ectópica.

\begin{abstract}
Adenomas are the most frequent hypophyseal tumors, and they can be occasionally located outside the sella turcica, either by growth from it or, less frequently, by ectopic location.

The case of a 56 year old female is presented, in which a brain TCprescribed in the context of a vertiginous syndrome revealed a lesion located in the sphenoid region extending to the clivus. Several diagnoses were discussed: chordoma, nasopharyngeal carcinoma, minor salivary gland derived tumor, and metastatic tumor. The lesion was surgically approached and excised, and it was finally demonstrated to be an ectopic hypophyseal adenoma.

Key words: Ectopic adenoma, ectopic hypophysis.
\end{abstract}

\footnotetext{
1 Médico del Hospital Clínico Fusat, Rancagua.

2 Médico del Servicio de Otorrinolaringología, Hospital San Juan de Dios, Universidad de Chile.
} 


\section{INTRODUCCIÓN}

El adenoma es el tumor hipofisiario más frecuente representando entre el $10 \%$ y $20 \%$ de todos los tumores cerebrales ${ }^{1}$. Ocasionalmente estos tumores pueden estar fuera de la silla turca ubicándose en la nariz, nasofaringe 0 seno esfenoidal, tales casos usualmente representan la extensión de una lesión masiva e invasiva intraselar (de hecho la cavidad nasal puede estar comprometida en más del $2 \%$ de los adenomas hipofisiarios) $)^{1,2}$, 0 más rara vez, ocurren en sitios ectópicos, es decir sin conexión con la glándula hipófisis intraselar (Coristoma).

Los adenomas hipofisiarios ectópicos son, por tanto, neoplasias muy raras, cuyo origen permanece como materia de debate, aunque lo más aceptado es que estos tumores se originan a lo largo del camino de migración de la bolsa de Rathke 0 en células pituitarias aberrantes dentro del tallo hipofisiario o la pars tuberalis ${ }^{3}$.

Desde su descripción original por Erdheim en 1909, alrededor de 50 casos de adenoma hipofisiario ectópico han sido comunicados ${ }^{4}$, de éstos el $62 \%$ se ha presentado en mujeres con un promedio de edad de 50,3 años, localizándose la mayoría de ellos a nivel del seno esfenoidal $(40 \%)$ o en la región supraselar $(33 \%)^{4}$. Otras localizaciones más esporádicas son el clivus, seno cavernoso, hueso petroso, hueso esfenoidal, tercer ventrículo, y hemisferio cerebral izquierd $0^{4,5}$. Si bien se trata de tumores benignos, éstos pueden presentarse como neoplasias agresivas que incluso generan parálisis de nervios craneales, siendo similares en inmunohistoquímica, morfología y actividad hormonal a los adenomas hipofisiarios típicos. Es así como un tercio de los adenomas hipofisiarios ectópicos comunicados en la literatura son endocrinológicamente inactivos, mientras que los dos tercios restantes presentan una actividad hormonal y usualmente secretan ACTH cursando por consiguiente con enfermedad de Cushing, pudiendo también asociarse a acromegalia e hiperprolactinemia.

Los signos y síntomas de presentación se relacionan con el efecto de masa y ocupación local (cefalea, obstrucción nasal) y/o con la hipersecreción hormonal (Cushing, acromegalia, hiperprolactinemia) $)^{1,5,6}$. Tanto la tomografía computada (TC) como la resonancia nuclear magnética (RNM) son muy útiles en el estudio de estas lesiones, siendo su diagnóstico preoperatorio difícil pues los procedimientos diagnósticos habitualmente sugieren otro tipo de neoplasias: cordomas, carcinoma nasofaríngeo, tumor derivado de una glándula salival menor 0 un tumor metastático; destacan dentro de los diagnósticos diferenciales más frecuentes. El diagnóstico histológico puede no ser difícil de hacer si el origen hipofisiario del tumor se conoce, sin embargo, cuando la biopsia inicial es tomada desde fuera de la fosa hipofisiaria, la posibilidad de adenoma hipofisiario puede no ser considerada.

El diagnóstico histológico diferencial incluye al tumor carcinoide, carcinoma neuroendocrino, paraganglioma, neuroblastoma olfatorio y carcinoma del tracto respiratorio superior. La transformación maligna de estos tumores es excepcional ${ }^{1,4} \mathrm{y}$ la terapia quirúrgica es el pilar del tratamiento, pudiendo asociarse a radioterapia posoperatoria cuando la resección es incompleta ${ }^{3,4}$.

\section{EMBRIOLOGÍA}

Embriológicamente la hipófisis se origina totalmente del ectodermo, a partir de dos porciones: 1. Una evaginación diencefálica hacia caudal (el Infundíbulo). 2. Una evaginación ectodérmica del estomodeo (cavidad bucal primitiva) anterior a la membrana bucofaríngea (la Bolsa de Rathke).

En la tercera semana de gestación, la bolsa de Rathke se evagina de la cavidad bucal y crece en

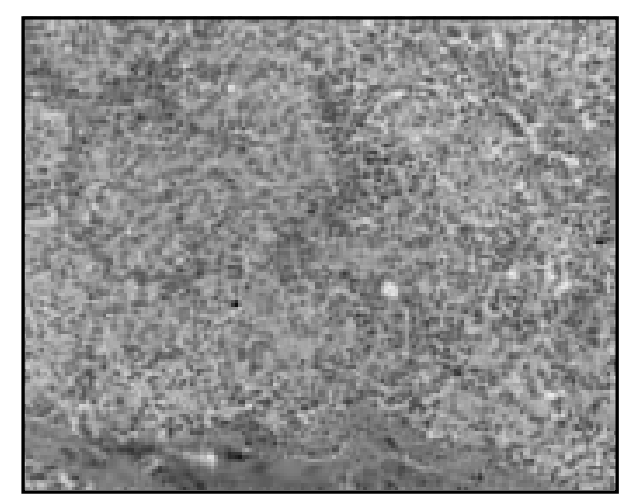

Figura 1. Proliferación neoplásica de hábito epitelial, normotípica, de núcleos redondos, discretamente hipercrómicos, algo irregulares en su contorno con citoplasma levemente eosinófilo, abundante. Conclusión: adenoma hipofisiario. 


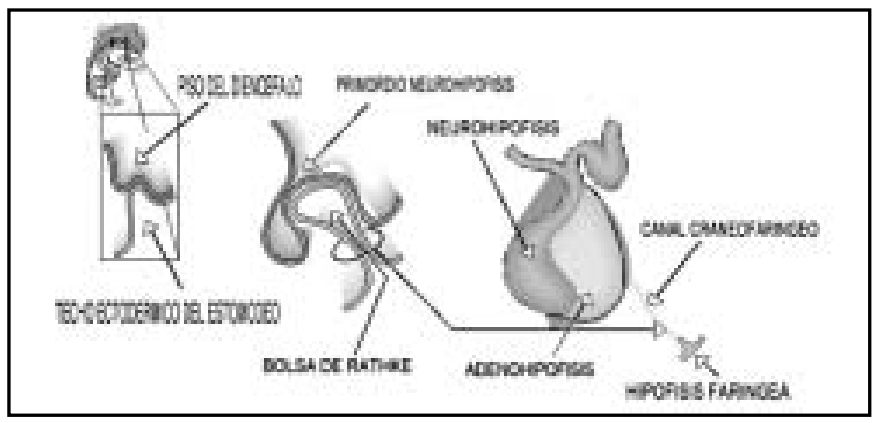

Figura 2. Desarrollo embriológico hipofisiario.

sentido dorsal hacia el infundíbulo, al final del $2^{\circ}$ mes pierde su conexión con la cavidad bucal y se halla en íntimo contacto con el infundíbulo, luego las células de la pared anterior de la bolsa de Rathke forman el lóbulo anterior de la glándula 0 adenohipófisis, una pequeña prolongación de este lóbulo, la pars tuberalis, crece a lo largo del tallo del infundíbulo rodeándolo y la parte posterior de la bolsa de Rathke se convierte en la pars intermedia mientras el infundíbulo da origen al tallo y a la pars nervosa 0 lóbulo posterior de la hipófisis (la neurohipófisis.).

La luz de la bolsa de Rathke se oblitera durante la formación y la conexión con la bucofaringe desaparece en una fase temprana del desarrollo debido al crecimiento del esfenoides, pero algunas pocas células en la porción inferior de la bolsa persisten a lo largo del trayecto, en ocasiones dentro del esfenoides, conociéndose como hipófisis faríngea; siendo estas células las que pueden dar origen a la formación de una tumoración hipofisiaria "ectópica"

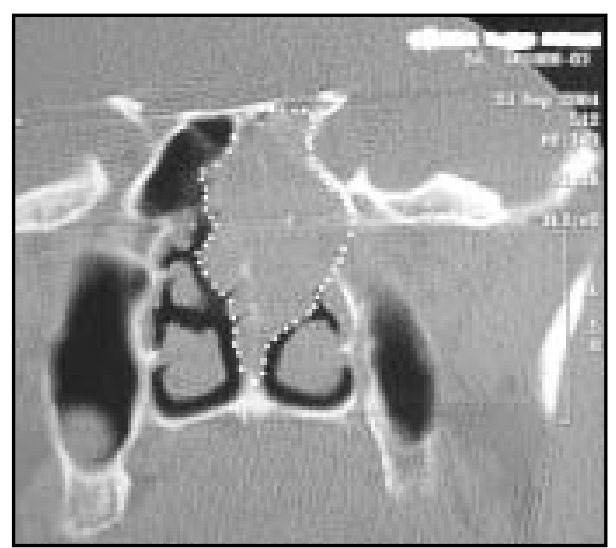

Figura 3

\section{CASO CLÍNICO}

Paciente de 56 años género femenino, hipertensa y obesa mórbida, diabética tipo 2 compensada, que consulta en julio de 2004 por un síndrome vertiginoso en el contexto del cual se solicita una TC de cerebro en la que se describe una silla turca aumentada de tamaño en diámetro anteroposterior y una ocupación esfenoidal asociada, sugiriéndose allí complementar el examen con una TC de silla turca en la que se encuentra una masa de aspecto tumoral, de predominio hipodenso que parece destruir el piso selar y parcialmente el clivus; se extiende desde región selar hacia caudal ocupando gran parte del seno esfenoidal en su aspecto izquierdo comprometiendo algunas celdillas etmoidales de ese lado. En resumen el informe concluye la presencia de una lesión expansiva que se extiende desde la silla turca hasta la cavidad nasal (Figuras 3 y 4 ).

Con estos hallazgos y para completar su estudio, se solicita una RNM (09/2004) que muestra

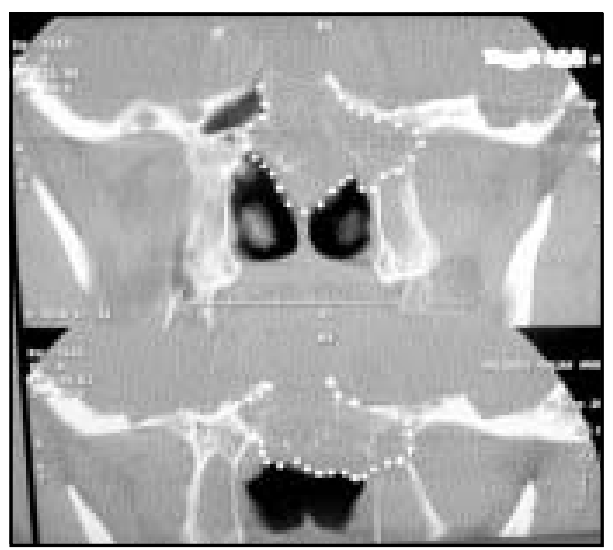

Figura 4

Figuras 3 y 4 . Cortes coronales de TAC de silla turca con lesión tumoral demarcada por punteado blanco. 
una lesión de base de cráneo centrada en el cuerpo del esfenoides y seno esfenoidal, con extensión a región nasoetmoidal y nasofaríngea especialmente a izquierda y en la parte baja de la fosa hipofisiaria; masa tenuemente hiperintensa en T1 en relación a estructuras musculares e hiperintensa moderada en T2. En el "Flair" presenta refuerzo algo heterogéneo luego del uso de medio de contraste que compromete el cuerpo del esfenoides, parte anterior del clivus, región del seno esfenoidal y parte anterosuperior de la región rinofaríngea así como la fosa nasal izquierda a nivel de la coana, la región nasoetmoidal izquierda y la parte posterior del tabique nasal (Figuras 5, 6 y 7 ).

El 23/10/2004 se realiza en el Hospital Clínico Fusat de Rancagua, cirugía con abordaje endoscópico y presencia de neurocirujanos en sala de operación, para acceder al área de la lesión se realiza una conchectomía media parcial derecha, extirpación de unciforme con etmoidectomía me- dia y posterior del mismo lado, extracción de la pared anterior del seno esfenoidal derecho que se observa normal; extirpación del rostrum del esfenoides apreciándose lesión de tipo quístico del esfenoides izquierdo que se extrae. Hacia inferior se observa masa de aspecto tumoral blanda que se fragmenta con facilidad al manipularla, cuya biopsia rápida fue informada como tumor de origen neural y cuya malignidad no logra precisarse mediante este estudio. Se completa la escisión de la lesión utilizando fresa diamantada sobre clivus. En el interior del seno esfenoidal, se observan paredes de éste conservadas sin dehiscencias ni comunicaciones hacia silla turca y sin evidencias de fístula de LCR al completar el procedimiento, con quiste de retención mucosa en su parte izquierda que en imagenología se observaba ocupada.

La biopsia diferida concluye la existencia de un adenoma hipofisiario, que dada su ubicación (sin conexión con hipófisis intraselar) sería ectópico.

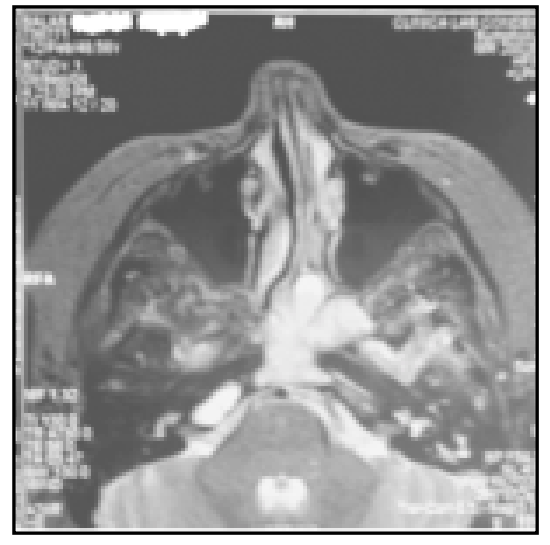

Figura 5.

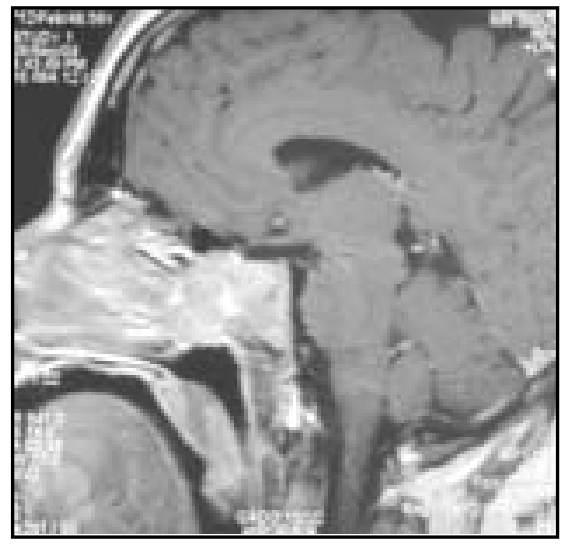

Figura 6.

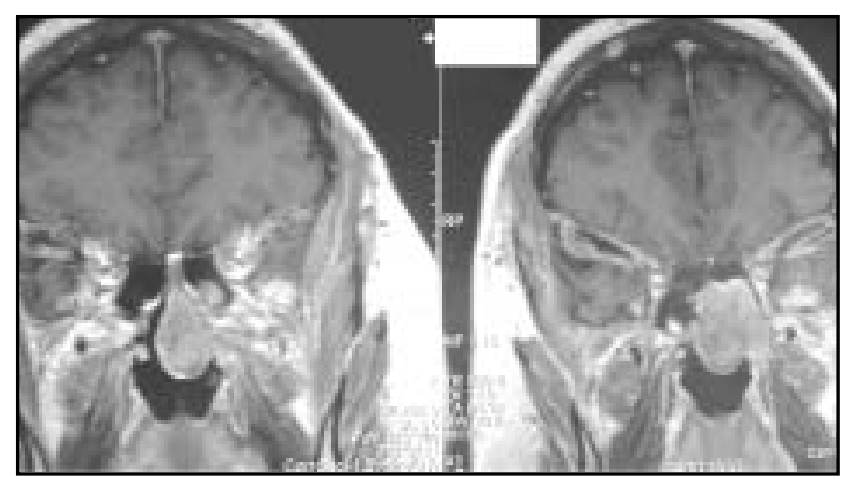

Figura 7. 
Evoluciona adecuadamente hasta su último control en octubre de 2007; sin signos de recidiva a los 3 años de la cirugía tanto al examen endoscópico como a la RNM (Figuras 11, 12 y 13, con sus contrapartes preoperatorias Figuras 8, 9 y 10). Exámenes endocrinológicos realizados posteriores a su cirugía resultaron normales.

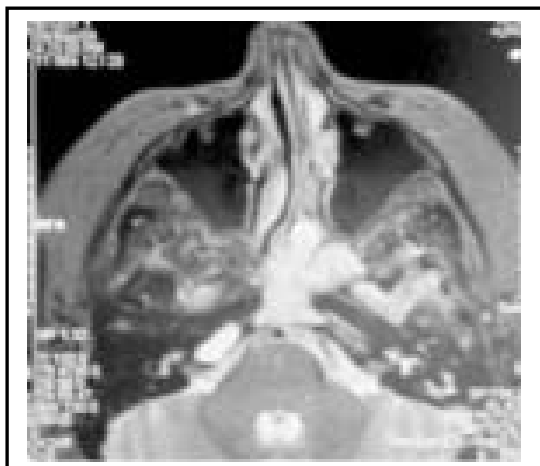

Figura 8

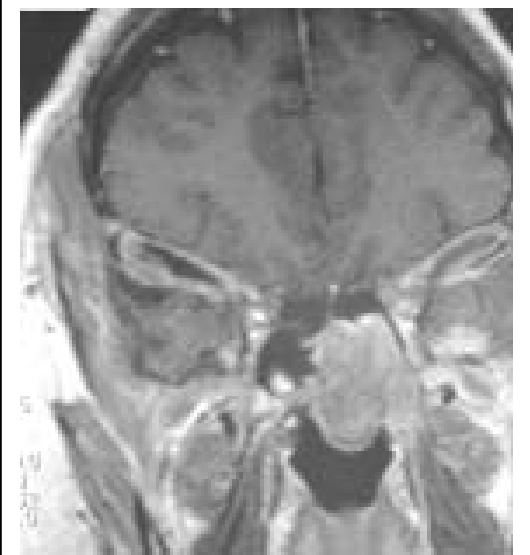

Figura 9

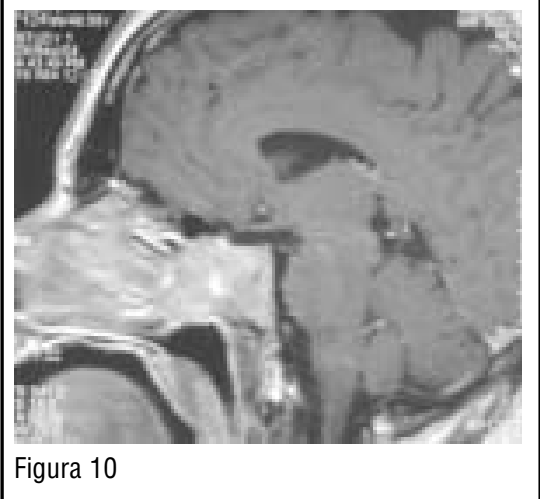

Figuras 8,9 y 10. RNM preoperatorias, axial, coronal y sagital respectivamente.

\section{COMENTARIO}

En resumen los adenomas hipofisiarios pueden presentarse como tumor sinonasal por invasión desde un tumor intrasellar o más rara vez por ocurrencia ectópica1. El caso descrito corresponde a un adenoma ectópico genuino pues no existía ninguna

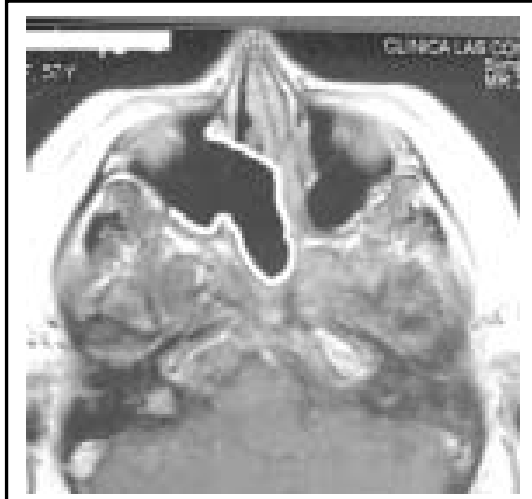

Figura 11

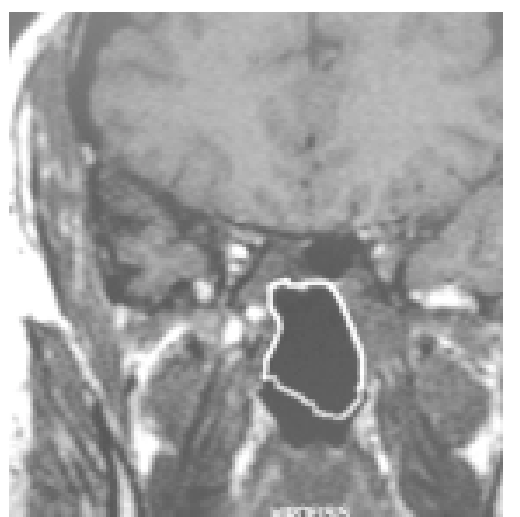

Figura 12

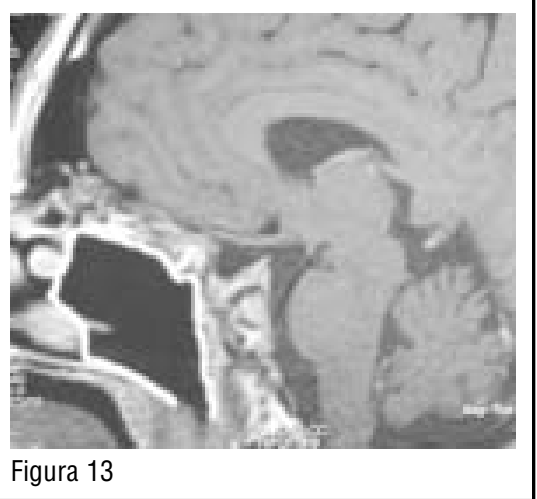

Figuras 11, 12 y 13. RNM posoperatorios de los mismos cortes anteriores con escisión quirúrgica demarcada con línea blanca. 
comunicación entre el tumor nasal encontrado y la glándula hipófisis, esto pudo ser comprobado sóolo en el intraoperatorio, pues imagenológicamente existía una aparente continuidad entre la lesión y la hipófisis, imagen generada por la ocupación esfenoidal que intraoperatoriamente se comprobó correspondía a un quiste de retención mucoso que respetaba las paredes del seno.

El alto índice de sospecha, la correlación con los hallazgos radiológicos y la inmunohistoquímica proyectada por marcadores neuroendocrinos y hormonas hipofisiarias específicas son útiles ayudas para llegar al diagnóstico correcto de este tipo de lesiones ${ }^{1,3,4}$.

\section{BIBLIOGRAFÍA}

1. Luk I, MRCPath, Chan J. Pituitary Adenoma Presenting as Sinonasal Tumor: Pitfalls in Diagnosis. Human Pathology 1996; 27: 605-609. A
2. Van Der Mey A, Van Krieken J, Dulken H, Van Seters A, VIelVOYe J, Hulshof J. Large Pituitary Adenomas With Extension into the Nasopharinx, report of three cases with a review of the literature. Ann Ool Rhinol Laryngol 1989; 98: 618-623. E

3.- LANGFord L, Batsakis J. Pituitary gland involvement of the sinonasal tract. Ann Otol Rhinol Laryngol 1995; 104: 167-9. C

4. Pasquini E, Faustini M, Sciarretta V, Saggese D, Roncaroli F, Serra D, Frank G. Ectopic TSHsecreting pituitary adenoma of the vomerosphenoidal juntion. European Journal of Endocrinology 2003; 148: 253-7. B

5. Lewis H, Todd H, Maie H, Victor T. Suprasellar Adrenocorticotropic Hormone-seccreting Ectopic Pituitary Adenoma: Case Report and Literature Review. Neurosurgery 2002; 50: 618-25. D

6. Collie, Brian R. Extracranial Thyroid-Stimulating Hormone-Secreting Ectopic Pituitary Adenoma of the Nasopharynx. Atolaryngol Head Neck Surg Volume 2005; 133: 453-4. 